\title{
Effects of the interplay between interaction and disorder in bipartite entanglement
}

\author{
L. F. Santos 1 , and G. Rigolin ${ }^{2,0}$ \\ ${ }^{1}$ Department of Physics and Astronomy, Michigan State University, East Lansing, MI 48824, USA \\ ${ }^{2}$ Departamento de Raios Cósmicos e Cronologia, \\ Instituto de Física Gleb Wataghin, Universidade Estadual de Campinas, \\ C.P. 6165, сер 13084-971, Campinas, São Paulo, Brazil
}

\begin{abstract}
We use a disordered anti-ferromagnetic spin- $1 / 2$ chain with anisotropic exchange coupling to model an array of interacting qubits. All qubits have the same level spacing, except two, which are called the defects of the chain. The level spacings of the defects are equal and much larger than all the others. We investigate how the entanglement between the two defects depends on the anisotropy of the system. When the anisotropy coupling is much larger than the energy difference between a defect and an ordinary qubit, the two defects become strongly entangled. Small anisotropies, on the contrary, may decrease the entanglement, which is, in this case, also much affected by the number of excitations. The analysis is made for nearest neighbor and next-nearest neighbor defects. The decrease in the entanglement for nearest neighbor defects is not very significant, especially in large chains.

PACS numbers: 03.67.Mn, 75.10.Jm
\end{abstract}

\section{INTRODUCTION}

Entanglement is one of the most striking properties of quantum mechanics. It describes a correlation between quantum mechanical systems that does not occur in classical mechanics, namely that a pure state of a composite quantum system cannot be written as a product of the states of its constituents [1]. It has long been a subject of interest in foundations of quantum mechanics, but recently it has acquired a fundamental role in quantum computation and quantum information [2].

Several attempts to quantify entanglement have been developed [3], but this is an area of research still in progress. In the case of a pure state of a bipartite system, a good and widely accepted measure of entanglement is the von Neumann entropy of its reduced density matrix. This entropy can be associated with a quantity called concurrence [4], which we adopt here as our measure of entanglement. The concurrence varies from 0 to 1 , the larger it is the more entangled is the state.

Spin chains are ideal systems for the study of entanglement. They are naturally used to model quantum computers (QC's): the two states of a spin- $1 / 2$ particle correspond to the two levels of a qubit and the exchange interaction corresponds to the qubit-qubit interaction. A particle with spin up corresponds to an excitation or an excited qubit. We consider a spin chain described by the $X X Z$ model. This is the model used in quantum computers based on electrons on helium 5]. The Ising part of the interaction is proportional to the anisotropy coupling, as shown in the next section. It only plays a role when two or more excitations are present in the chain. The XY part of the interaction is responsible for hopping

\footnotetext{
*Electronic address: santos@pa.msu.edu
}

${ }^{\dagger}$ Electronic address: rigolin@ifi.unicamp.br the excitations and can be used to create entanglement between two or more qubits [6, 7]. However it can also quickly destroy bipartite entanglement, because the excitations soon spread all over the chain.

A way to avoid the spreading (or delocalization) of the excitations is by tuning the qubits away from resonance [8]. Such control of the qubit level spacings enables us to entangle just some specific qubits. This is the strategy we use in this paper. To have two chosen qubits maximally entangled, we tune them in resonance. Their level spacings are different from all other qubits, they are the defects of the chain.

When there is only one excitation in the chain, the entanglement between the two selected sites becomes trivial. By assuming that the energy difference between the defects and the ordinary qubits is much larger than the strength of the $X Y$ interaction, the Hamiltonian of the system gives two maximally entangled states. They correspond to superpositions of the basis states where the excitation occupies one of the defects [7]. However, when more than one excitation is present, the entanglement between the defects can be significantly affected by the Ising interaction. The main purpose of the present work is to analyze which is, in this case, the eigenstate with maximum concurrence and how the value of the maximum concurrence depends on the anisotropy.

We find numerically that for large values of the anisotropy coupling, nearest neighbor defects are strongly entangled and the value of the concurrence is almost negligibly affected by the number of excitations in the system. In the case of separated defects, this observation is valid only for long chains. In the opposite situation of small anisotropies, the concurrence between the defects is strongly dependent on the number of excitations and it can become quite small. Partial analytical justifications for these observations are provided.

The paper is organized as follows. In Sec. II we describe the model and the adopted measure for bipartite 
entanglement. In Sec. III we analyze the maximum concurrence between nearest neighbor and next nearest neighbor defects for various values of the anisotropy coupling. A discussion on how to prepare maximally entangled states and how the anisotropy may help is also presented in this section.

\section{THE MODEL AND THE MEASUREMENT OF ENTANGLEMENT}

We consider a spin chain with nearest neighbor interaction. The Hamiltonian describing the system is given by the $X X Z$ model

$$
\begin{aligned}
& H=\sum_{n=1}^{L} \frac{\varepsilon_{n}}{2} \sigma_{n}^{z}+\frac{J}{4} \sum_{n=1}^{L}\left[\Delta \sigma_{n}^{z} \sigma_{n+1}^{z}+\frac{1}{2} H_{\mathrm{hop}}\right], \\
& H_{\mathrm{hop}}=\left(\sigma_{n}^{+} \sigma_{n+1}^{-}+\sigma_{n}^{-} \sigma_{n+1}^{+}\right),
\end{aligned}
$$

where $\hbar=1$ and $\sigma^{z,+,-}$ are Pauli matrices. There are $L$ sites and we deal with a periodic (or closed) chain, that is, sites $n+L$ and $n$ are the same. Each site $n$ is subjected to a magnetic field in the $z$ direction, giving the energy splitting $\varepsilon_{n}$. In this description, the excitation energy of a qubit is the Zeeman energy of a spin. In this disordered chain, not all the qubits have the same level spacing $\varepsilon$. The energy of two qubits, called defects, differs from those of other qubits by $d$. The parameter $J$ is the hopping integral and $\Delta$ is a dimensionless parameter related to the anisotropy coupling. The diagonal term $\sigma_{n}^{z} \sigma_{n+1}^{z}$ gives the Ising interaction and the non-diagonal term $H_{\text {hop }}$ is responsible for propagating the excitations. We set $d, J$ and $\Delta>0$.

The anisotropy in the Hamiltonian (11) is different from the one considered in some previous models $[9,10,11$. The hopping part of the Hamiltonian can be equivalently written as $H_{\text {hop }} \propto J_{x} \sigma_{n}^{x} \sigma_{n+1}^{x}+J_{y} \sigma_{n}^{y} \sigma_{n+1}^{y}$. Here $J_{x}=J_{y}=J$, but in the models cited above, the degree of anisotropy comes from the difference between $J_{x}$ and $J_{y}$. The anisotropy in our case originates from the extra Ising interaction. In terms of entanglement, few studies have been developed with this model [12, 13]. Moreover, our goal is not simply to analyze how entanglement may depend on the described anisotropy, but we aim to analyze how it is affected by the interplay between this interaction and disorder.

The disorder of the system we consider is characterized by the presence of the two defects. In principle, total control of the qubit level spacings is available, which allows the creation of defects.

The Ising part of the $X X Z$ model combined with defects has been used before to create maximally entangled states []. The study of entanglement with impurities, but without any $\sigma_{n}^{z} \sigma_{n+1}^{z}$ interaction, has also been done in 10, 11]. We emphasize the importance of studying the effects of this extra interaction in disordered systems, which are far from trivial. The Ising interaction is only relevant when at least two excitations are present, being therefore associated with many-body problems. It is actually at the heart of one of the most challenging problems in condensed matter physics, namely the difficulty in localizing many-particle states [8].

In what follows, we count energy off the ground state energy $E_{0}=-(L \varepsilon+2 d) / 2+L J \Delta / 4$, i.e., we replace in Eq. (11) $H \rightarrow H-E_{0}$.

To address the different states of the system we use a notation that is common in the study of spin chains with the Bethe ansatz [14]. The state corresponding to one single excitation on site $n$, that is $\mid \downarrow_{1} \downarrow_{2} \ldots \downarrow_{n-1} \uparrow_{n} \downarrow_{n+1}$ $\left.\ldots \downarrow_{L}\right\rangle$, or equivalently $\left|0_{1} 0_{2} \ldots 0_{n-1} 1_{n} 0_{n+1} \ldots 0_{L}\right\rangle$, is simply written as $\phi(n)$. The state of two excitations, one on site $n$ and the other one on site $m$, is $\phi(n, m)$, which is a simplified notation for $\mid \downarrow_{1} \downarrow_{2} \ldots \uparrow_{n} \downarrow_{n+1} \quad \ldots \uparrow_{m} \quad \ldots \downarrow_{L}$ \rangle , or equivalently $\left|0_{1} 0_{2} \ldots 1_{n} 0_{n+1} \ldots 1_{m} \ldots 0_{L}\right\rangle$. Basis states where each excitation is confined to a single site, such as $\phi(n, m)$, are called in quantum computing quantum registers. These are the states where the measurements are performed.

Since we want to study the effects of the Ising interaction, several excitations have to be considered, which limits the numerical analysis to small chains. In the model described by Eq. (11), the $z$ component of the total spin $\sum_{n=1}^{L} S_{n}^{z}$ is conserved, so states with different number of excitations are not coupled and the Hamiltonian is made of uncoupled blocks. However the blocks can still be very large. For a chain with $L$ sites, the block corresponding to $N$ excitations has dimension $L ! /[N !(L-N)$ !]. Its diagonalization leads to eigenstates which correspond to linear superpositions of quantum registers $\phi$ with $N$ excitations. Each state $k$ is written as

$$
\left|\psi_{N, L}^{(k)}\right\rangle=\sum_{n<m<\ldots<N=1}^{L} a^{(k)}(n, m, \ldots, N)|\phi(n, m, \ldots, N)\rangle .
$$

In order to study quantitatively the entanglement between two qubits we calculate their entanglement of formation $E_{F}$. Given the density matrix $\rho$ that describes our pair of qubits, $E_{F}$ is the average entanglement of the pure states of the decomposition of $\rho$, minimized over all possible decompositions:

$$
E_{F}(\rho)=\min \sum_{i} p_{i} E\left(\psi_{i}\right),
$$

where $\sum_{i} p_{i}=1,0<p_{i} \leq 1$, and $\rho=\sum_{i} p_{i}\left|\psi_{i}\right\rangle\left\langle\psi_{i}\right|$. Here $E(\psi)$ is the von Neumann entropy of either of the two qubits [15]. Wootters et al [4] have shown that, for a pair of qubits, $E_{F}$ is a monotonically increasing function of the concurrence, which one can prove to be an entanglement monotone. Since the concurrence is mathematically simpler to deal with than $E_{F}$, we adopt it here to measure the entanglement between two qubits. It is given by [4]:

$$
C=\max \left\{\lambda_{1}-\lambda_{2}-\lambda_{3}-\lambda_{4}, 0\right\},
$$


where $\lambda_{1}, \lambda_{2}, \lambda_{3}$, and $\lambda_{4}$ are the square roots of the eigenvalues, in decreasing order, of the matrix $R=\rho \tilde{\rho}$. The matrix $\tilde{\rho}$ is the time reversed matrix

$$
\tilde{\rho}=\left(\sigma_{y} \otimes \sigma_{y}\right) \rho^{*}\left(\sigma_{y} \otimes \sigma_{y}\right) .
$$

The symbol $\rho^{*}$ means complex conjugation of the matrix $\rho$ in the basis $\{|11\rangle,|10\rangle,|01\rangle,|00\rangle\}$.

Maximum entanglement corresponds to $C=1$ and no entanglement gives $C=0$. To compute the concurrence of two qubits in a chain with several sites, we trace over the qubits we are not interested in and study the reduced density matrix of the two chosen ones.

\section{NUMERICAL RESULTS AND ANALYTICAL ANALYSIS}

We study the entanglement between the two defects of the chain. The difference in energy between them and the other qubits, $d$, is assumed much larger than the hopping integral, $d>>$. This guarantees that an excitation placed on one defect can only hop between the two defect sites. As a consequence, among all the eigenstates of the system, the ones with a single excitation shared between the defects have the largest concurrences.

In the first subsection below, we analyze the case where the defects correspond to two nearest neighbor qubits and in the second subsection they are two next nearest neighbors. Since the chain has periodic boundary conditions, any pair of qubits $\left(n_{0}, m_{0}\right)$ translated through the chain is equivalent, so we choose, for the numerical calculations, the pair 1 and 2 as nearest neighbor defects and the pair 1 and 3 as next-nearest neighbor defects.

We study how the maximum concurrence between the defects depend on the anisotropy and which is the corresponding eigenstate.

\section{A. Nearest neighbor defects}

The numerical results for the dependence of the maximum concurrence, $C_{\max }$, on the anisotropy of a periodic Heisenberg chain with two neighbor defects are shown in Fig. 11 When $\Delta=0$, all states with one excitation on the defects have $C_{\max } \simeq 1$. For small $\Delta$ 's, in general, $C_{\max }$ decreases with the number of excitations. The comparison of $C_{\max }$ for chains of different sizes but with the same number of excitations indicates that, in most cases, smaller chains are more affected by the Ising interaction. When there are just two excitations, the minimum value of $C_{\max }$ happens when $J \Delta=d$, while in the case of more excitations, this occurs for smaller values of $\Delta$. Whenever $J \Delta \gg d$, the maximum concurrence stabilizes in a value close to 1 (the larger the chain the closer to 1 it will be). In this case, the dependence on the number of excitations becomes little noticeable. In what follows, we try to find justifications, some times analytically, to these observations.

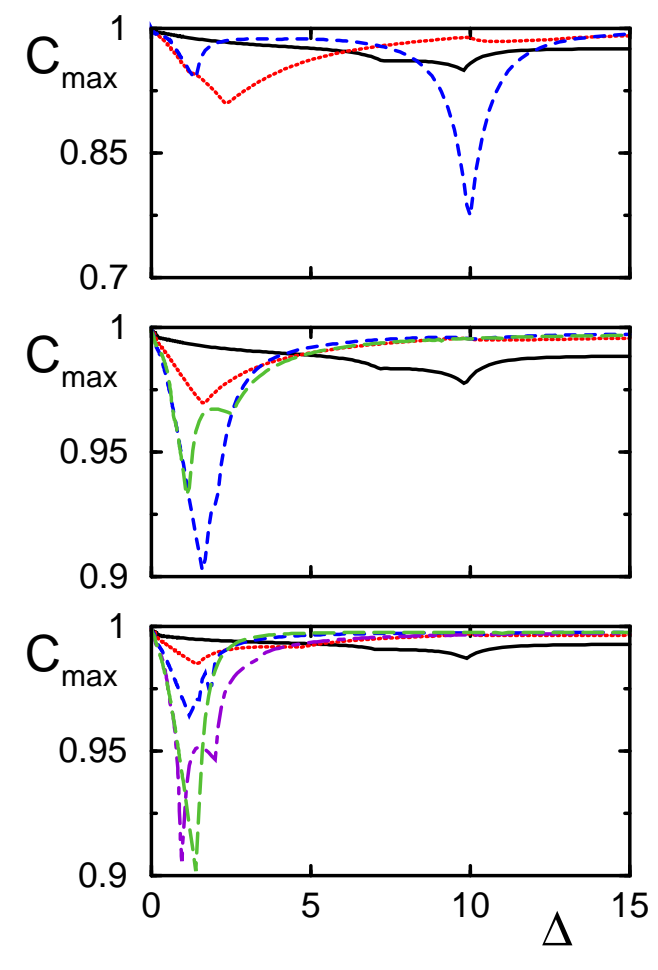

FIG. 1: (color online) Maximum concurrence vs. anisotropy coupling in closed Heisenberg spin- $1 / 2$ chains with defects located on sites 1 and 2 . The energy difference between the defects and the other qubits is $d=10 \mathrm{~J}$. The panels correspond to data for chains of different lengths: $L=8$ (top), $L=10$ (middle) and $L=12$ (bottom). The maximum number of excitations considered for each chain is $L / 2$. The curves are chosen as follows: solid (black) curves for 2 excitations, dotted (red) for 3 excitations, dashed (blue) for 4 excitations, long dashed (green) for 5 excitations, and dot-dashed (violet) for 6 excitations.

The eigenvalues and eigenstates of an anisotropic spin chain with no defects can be analytically obtained with the Bethe ansatz [14]. In the presence of defects, where $d \sim J$, most commonly the chain becomes non integrable [16, 17]. When $d \gg J$, as in this paper, we are again capable of solving the eigenvalue problem in certain situations. This happens because, with such large $d$, the $X Y$-type interaction between defect and ordinary qubit is negligible, there is no hopping of excitations between them. It works as if we had cut the closed chain and created two chains with free boundaries (two open chains): a small one corresponding simply to the two defects and another one with $L-2$ sites.

In the simple case of a single excitation in the chain, the spectrum is divided into two well separated bands. One is made of $L-2$ states with energy in the range $\varepsilon_{1}-J \leq E_{1} \leq \varepsilon_{1}+J$, where $\varepsilon_{1}=\varepsilon-J \Delta$. They are states with the excitation out of the defects. The other band is made of just the following two linear superpositions $\left[\phi\left(n_{0}\right) \pm \phi\left(n_{0}+1\right)\right] / \sqrt{2}$, with energy $\varepsilon_{1}+d \pm J / 2$, respectively. They correspond to Bell (or EPR) states 
and have concurrence equal to 1 .

In this paper we want to analyze how the Ising interaction affects the entanglement and so we consider two or more excitations.

\section{Two excitations and $\Delta=0$}

In the case of two excitations, but with $\Delta=0$, we should obtain results similar to ones for one excitation, that is the states with maximum concurrence should give $C_{\max }=1$, since no Ising interaction exists. The states of maximum concurrence between the defect sites have one excitation shared between them. They are superpositions of the quantum registers with energy $\varepsilon_{2}+d$, where $\varepsilon_{2}=$ $2 \varepsilon-2 J \Delta$ and form an energy band that we will call defect band, or $d$-band for short. The transitions between these quantum registers are schematically shown below. Each quantum register (apart from the border ones) is coupled to three others: two corresponding to an excitation hop to an ordinary qubit and one associated to an excitation hop to the other defect.

$$
\begin{aligned}
& n_{0}, n_{0}+2 \quad \leftrightarrow \quad n_{0}, n_{0}+3 \quad \leftrightarrow \quad \ldots \quad n_{0}, n_{0}+L-1 \\
& \uparrow \quad \uparrow \quad \uparrow \\
& n_{0}+1, n_{0}+2 \leftrightarrow n_{0}+1, n_{0}+3 \leftrightarrow \ldots n_{0}+1, n_{0}+L-1
\end{aligned}
$$

The states of the $d$-band have the form

$$
\begin{aligned}
\left|\psi_{2, L}^{\left(k_{1}, k_{2}\right)}\right\rangle & =\sum_{n, m} c(n, m)|\phi(n, m)\rangle \\
& =\sum_{m=n_{0}+2}^{n_{0}-1+L} c\left(n_{0}, m\right)\left|\phi\left(n_{0}, m\right)\right\rangle \\
& +\sum_{m=n_{0}+2}^{n_{0}-1+L} c\left(n_{0}+1, m\right)\left|\phi\left(n_{0}+1, m\right)\right\rangle .
\end{aligned}
$$

[To avoid a heavy notation, the superscript $\left(k_{1}, k_{2}\right)$ was suppressed from the right hand side.].

We use the Bethe ansatz method to find the coefficients $c(n, m)$ for these $2(L-2)$ states. The Schrödinger equation for $c(n, m)$ is

$$
\begin{aligned}
E_{2} c(n, m) & =\left(\varepsilon_{2}+d\right) c(n, m)+\frac{J}{2}[c(n-1, m) \\
& +c(n+1, m)+c(n, m-1)+c(n, m+1)] .
\end{aligned}
$$

Since the studied chain became equivalent to two open chains, it is natural to write

$$
\begin{aligned}
& c(n, m)=a(n) b(m)= \\
& {\left[A_{1} \mathrm{e}^{\mathrm{i} \alpha n}+A_{2} \mathrm{e}^{-\mathrm{i} \alpha n}\right]\left[B_{1} \mathrm{e}^{\mathrm{i} \beta m}+B_{2} \mathrm{e}^{-\mathrm{i} \beta m}\right] .}
\end{aligned}
$$

The energy $E_{2}$ as a function of $\alpha$ and $\beta$ is obtained from (7), (8) and has the form

$$
\begin{aligned}
& E_{2}=\varepsilon_{2}+d+E_{a}+E_{b}, \\
& E_{a}=J \cos \alpha, \\
& E_{b}=J \cos \beta .
\end{aligned}
$$

Using the boundary conditions for the small chain of defects

$$
a\left(n_{0}-1\right)=a\left(n_{0}+2\right)=0,
$$

and the boundary conditions for the chain of ordinary qubits

$$
b\left(n_{0}+1\right)=b\left(n_{0}+L\right)=0
$$

we obtain

$$
c(n, m)=A \sin \left[\alpha\left(n-n_{0}+1\right)\right] \sin \left[\beta\left(m-n_{0}-1\right)\right],
$$

where $A$ is a normalization constant and $\alpha$ and $\beta$ are given by

$$
\begin{aligned}
& \alpha=\pi k_{1} / 3, \quad k_{1}=1,2 \\
& \beta=\pi k_{2} /(L-1), \quad k_{2}=1,2, \ldots, L-2 .
\end{aligned}
$$

Each one of the $2(L-2)$ states have then a "Bell-type" form

$$
\begin{aligned}
& \left|\psi(n, m)^{\left(k_{1}, k_{2}\right)}\right\rangle=\sum_{m=n_{0}+2}^{n_{0}-1+L} A \sin \left[\frac{\pi k_{2}\left(m-n_{0}-1\right)}{(L-1)}\right] \\
& \times \frac{1}{\sqrt{2}}\left[\phi\left(n_{0}, m\right)+(-1)^{k_{1}+1} \phi\left(n_{0}+1, m\right)\right] .
\end{aligned}
$$

To calculate the concurrence between the two defects, we obtain the reduced density matrix of the states above by tracing over the ordinary qubits. Only the four elements in the middle are different from zero. We have

$$
\rho=\left(\begin{array}{cccc}
0 & 0 & 0 & 0 \\
0 & 1 / 2 & (-1)^{k_{1}+1} / 2 & 0 \\
0 & (-1)^{k_{1}+1} / 2 & 1 / 2 & 0 \\
0 & 0 & 0 & 0
\end{array}\right)
$$

Therefore, when $\Delta=0$, all the states in the $d$-band have concurrence equal to 1 . These analytical results agree very well with the numerical ones.

\section{Two excitations and $0<\Delta<d / J$}

The situation changes as we start increasing $\Delta$. The scheme for the involved transitions from the previous sub-subsection still applies, but the quantum registers $\phi\left(n_{0}-1, n_{0}\right)$ and $\phi\left(n_{0}+1, n_{0}+2\right)$ now have on-site energy equal to $\varepsilon_{2}+d+J \Delta$, which is larger than the energy 
of the other quantum registers considered. As a consequence, the coefficients $c(n, m)$ are not simply written as the product (8), which leads to the "Bell-type" states given by Eq.(14).

However, Eq. (8) can still be a reasonable approximation to obtain the state with the lowest energy in the $d$ band. This state necessarily has small coefficients for the exceptional registers $\phi\left(n_{0}-1, n_{0}\right)$ and $\phi\left(n_{0}+1, n_{0}+2\right)$, so the effects caused by their different energies, which move the eigenstates away from the "Bell-type" states, are not so significant. Such "ground" state of the band has, as expected and also confirmed numerically, the largest concurrence. It can be found analytically by using Eqs.(8), (10), and some new boundary conditions for the chain of ordinary qubits. This is a relatively good approximation to the actual state and provides a good description for the decay of $C_{\max }$ with $\Delta$.

Since we are interested in the $d$-band ground state, we select $k_{1}=2$, which gives the smallest $\cos \alpha$. The boundary conditions for the coefficients $b(m)$ are now different. When the first excitation is on site $n=n_{0}$, the second excitation, necessarily out of the defects, has to satisfy

$$
b\left(n_{0}+1\right)=0 .
$$

Moreover, in this case, the second excitation is also subjected to the condition

$$
J \Delta b\left(n_{0}-1+L\right)+\frac{J}{2}\left[b\left(n_{0}-2+L\right)\right]=E_{b} b\left(n_{0}-1+L\right),
$$

which, when combined with the general equation,

$$
\frac{J}{2}[b(m-1)+b(m+1)]=E_{b} b(m),
$$

leads to

$$
J \Delta b\left(n_{0}-1+L\right)=\frac{J}{2} b\left(n_{0}+L\right)
$$

Equivalently, when the first excitation is on site $n=$ $n_{0}+1$, the boundary conditions for the second one are

$$
\begin{aligned}
& J \Delta b\left(n_{0}+2\right)=\frac{J}{2} b\left(n_{0}+1\right), \\
& b\left(n_{0}+L\right)=0 .
\end{aligned}
$$

The wave function in the case of $\Delta<d / J$ is then written as

$$
\begin{aligned}
|\psi(n, m)\rangle= & A \sum_{m=n_{0}+2}^{n_{0}-1+L}\left[S\left(n_{0}+L-m\right) \phi\left(n_{0}, m\right)\right. \\
& \left.+S\left(m-n_{0}-1\right) \phi\left(n_{0}+1, m\right)\right]
\end{aligned}
$$

where A is a normalization constant and the function $S(x)$ is given by

$$
S(x)=2 \Delta \sin [\beta(x-1)]-\sin [\beta x] .
$$

The $L-2$ different and non-trivial values of $\beta$ are obtained from

$$
2 \Delta \sin [\beta(L-2)]=\sin [\beta(L-1)] .
$$

We notice once again that we are only interested in the value of $\beta$ that gives the smallest $\cos \beta$. The eigenvalues obtained with Eqs.(9), (12), and (23) are fairly similar to the ones obtained numerically, but the results for the eigenvectors (21) are so not good. The approximation used here is only acceptable for the lowest-energy state of the $d$-band. Its reduced density matrix becomes

$$
\rho=\left(\begin{array}{cccc}
0 & 0 & 0 & 0 \\
0 & 1 / 2 & |A|^{2} s & 0 \\
0 & |A|^{2} s & 1 / 2 & 0 \\
0 & 0 & 0 & 0
\end{array}\right)
$$

where

$$
s=\sum_{m=n_{0}+2}^{n_{0}-1+L} S\left(n_{0}+L-m\right) S\left(m-n_{0}-1\right) .
$$

The maximum concurrence obtained,

$$
\begin{aligned}
C_{\max }= & \sqrt{|A|^{4} s^{2}+|A|^{2} s+1 / 4} \\
& -\sqrt{|A|^{4} s^{2}-|A|^{2} s+1 / 4}
\end{aligned}
$$

is clearly smaller than $1 .|A|^{2} s$ can only be equal to $1 / 2$ when $\Delta=0$ and $\left|S\left(n_{0}+L-m\right)\right|=\left|S\left(m-n_{0}-1\right)\right|$, that is when all quantum registers forming the $d$-band are in resonance. However, $S\left(n_{0}+L-m\right)$ and $S\left(m-n_{0}-\right.$ 1) differ mostly at the borders of the chain of ordinary qubits (where $m=n_{0}+2$ or $m=n_{0}+L-1$ ) and this difference increases as $\Delta$ increases.

In the case of 8 sites, $d / J=10$ and $\Delta=3$, for example, we find $C_{\max } \sim 0.91$, while numerically we have $C_{\max } \sim 0.98$. For a longer chain with 12 sites and the same parameters, $C_{\max } \sim 0.97$, while numerically we have $C_{\max } \sim 0.99$. The agreement between numerics and analytics are not excellent, but the approximation gives the correct trend: the concurrence decreases as $\Delta$ increases (with $\Delta<d / J$ ), and the effects become less important in larger chains.

\section{Two excitations and $\Delta=d / J$}

In the case of resonance, when $\Delta=d / J$, the quantum registers $\phi\left(n_{0}+L-1, n_{0}\right)$ and $\phi\left(n_{0}+1, n_{0}+2\right)$ 
have very large energy and do not participate in the formation of the eigenstates in the defect band anymore. However, quantum registers corresponding to the bound pairs $\phi\left(n_{0}-2, n_{0}-1\right)$ and $\phi\left(n_{0}+2, n_{0}+3\right)$ now play an important role, for they are in resonance with the other registers and couple with them in first order of perturbation theory in $J$. As a result, a transition from the sites $\left(n_{0}+1, n_{0}+3\right)$ to $\left(n_{0}+2, n_{0}+3\right)$ [equivalently from $\left(n_{0}-2, n_{0}\right)$ to $\left.\left(n_{0}-2, n_{0}-1\right)\right]$, which removes the excitation from the defect, can happen. This is the cause of the drop in the value of the maximum concurrence.

The scattering process is schematically shown below. There are four states out of the core that could lead to a Bell-type state.

$$
\begin{array}{cccc} 
& & n_{0}-1, n_{0}+L-2 \\
n_{0}, n_{0}+2 & \leftrightarrow n_{0}, n_{0}+3 \leftrightarrow & \ldots & n_{0}, n_{0}+L-2 \\
\uparrow & & \uparrow \\
& n_{0}+1, n_{0}+3 \leftrightarrow & \ldots & n_{0}+1, n_{0}+L-2 \leftrightarrow n_{0}+1, n_{0}+L-1 \\
\uparrow & &
\end{array}
$$

Notice that even though the other bound pairs far from the defects are also in resonance with the above states, the coupling with them occurs in second order in $J$, so they form a separated band. A more thorough discussion can be found in Ref. [18], but the idea is the following. There are two possible channels for the second order coupling between distant bound pairs and the $d$-band states. A possibility is a transition from sites $\left(n_{0}+3, n_{0}+4\right)$ to $\left(n_{0}+1, n_{0}+4\right)$, but we can equivalently hop from $\left(n_{0}+3, n_{0}+4\right)$ to the bound pair next to the defect $\left(n_{0}+2, n_{0}+3\right)$ [two channels also exist for the excitations on sites $\left.\left(n_{0}-3, n_{0}-2\right)\right]$. The amplitudes for these two transitions are equal in magnitude, but opposite in sign. Due to such quantum interference, or antiresonance, we have two uncoupled bands of very close energy.

The antiresonance explains why the drop in the value of the maximum concurrence when $\Delta=d / J$ is not so drastic as one might have expected. Also, since there are only four states preventing the creation of a perfect Bell state, the decay of $C_{\max }$ becomes less perceptible in larger chains.

\section{Two excitations and $\Delta \gg d / J$}

When $\Delta \gg d / J$, the allowed transitions, shown below, now have two registers out of the core that could lead to a Bell-type state.

$$
\begin{aligned}
& n_{0}, n_{0}+2 \leftrightarrow n_{0}, n_{0}+3 \leftrightarrow \quad \ldots \quad n_{0}, n_{0}+L-2 \\
& \uparrow \\
& n_{0}+1, n_{0}+3 \leftrightarrow \quad \ldots \quad n_{0}+1, n_{0}+L-2 \leftrightarrow n_{0}+1, n_{0}+L-1
\end{aligned}
$$

This justifies why the maximum concurrence never goes back to 1 , even for very large $\Delta$. However, the effects caused by just these two isolated registers, $\phi\left(n_{0}, n_{0}+2\right)$ and $\phi\left(n_{0}+1, n_{0}+L-1\right)$, become less significant in larger chains.

\section{More than two excitations and $\Delta \gg d / J$}

When more than two excitations are present, the analysis of the maximum concurrence for small $\Delta$ 's gets more complicated. A more interesting and general situation emerges when $\Delta \gg d / J$. Here, we have again Bell-type states similar to the ones given by Eq. (14). These states have one excitation hopping between the two defects and all the others bound together in a cluster in sites far from the defects.

Only clusters with the same number of excitations are coupled and the transitions between them happen in a high order of perturbation theory in $J$. These transitions involve virtual steps where a dissociation occurs. Therefore the clusters move together as a whole and very slowly. We have found that states involving clusters at least two sites away from the defects have the largest concurrences. They are written as

$$
\begin{aligned}
& \left|\psi\left(n, m_{1}, m_{2}, \ldots, m_{N-1}\right)\right\rangle=\sum_{m_{1}=n_{0}+4}^{n_{0}+L-N-1} A \sin \left[\frac{\pi k_{2}\left(m_{1}-n_{0}-3\right)}{(L-N-3)}\right] \times \\
& \frac{1}{\sqrt{2}}\left[\phi\left(n_{0}, m_{1}, m_{1}+1, \ldots, m_{1}+N-2\right)+(-1)^{k_{1}+1} \phi\left(n_{0}+1, m_{1}, m_{1}+1, \ldots, m_{1}+N-2\right)\right],
\end{aligned}
$$

where $k_{1}=1,2$ and $k_{2}=1,2, \ldots, L-N-4$.

Notice that the states above cannot be obtained when we have small chains with $L \leq 8,9$ and the total number of excitations are $N=L / 2, L / 2+1$ respectively, as the available $L-6$ sites are not enough for the remaining $N-1$ excitations. But, for these small chains and also for larger ones, there are other sorts of Bell-type states that can be obtained making use of the anisotropy. An example is the state $1 / \sqrt{2}\left[\phi\left(n_{0}, n_{0}+2, n_{0}+3, n_{0}+L-\right.\right.$ $\left.\left.2, n_{0}+L-1\right)+\phi\left(n_{0}+1, n_{0}+2, n_{0}+3, n_{0}+L-2, n_{0}+L-1\right)\right]$ found when $L=10$. We also note that, in the case of $L=12$ and $N=5$, the states with large concurrences obtained numerically did not correspond to what was expected from (27), though they were also Bell states.

As a result, the anisotropy coupling may be harmful when small, but very useful when sufficiently large, for 
we can recover Bell-type states.

In the limit of very large $\Delta$ we can use the formation of clusters to create maximally entangled states that will remain as such for a long time. As an illustration, we take the case of $L=12,4$ excitations, $d / J=10$, $\Delta=50$ and the two defects placed on sites 1 and 2 . The most straightforward method to study the dynamics of the system consists of diagonalizing the $495 \times 495$ Hamiltonian (11) for the chosen parameters. We then assume that the initial state is the quantum register $\phi(1,6,7,8)$ with one excitation on site 1 and the other three excitations bounded in a cluster on sites 6,7 , and 8 , which is not an eigenstate of the Hamiltonian $H$. Its evolution is obtained by writing it as a linear superposition of the eigenstates of $H$ with their respective energies. The results are shown in Fig. 2 .

The excitation on site 1 hops between the two defects with a period $2 \pi / J$ [7]. The analytical reason for this is the following. The states with one excitation on one of the defects and three excitations bounded together form their own energy band around the value $\varepsilon_{4}+d+J \Delta$. These states are not coupled with the other states of the system and can be treated separately, largely reducing the Hamiltonian to be considered. In first order of perturbation theory, the Hamiltonian is still further reduced to a simple $2 \times 2$ matrix written in the basis of the two registers $\phi(1,6,7,8)$ and $\phi(2,6,7,8)$, which are coupled in first order. The off diagonal elements of this matrix are $J / 2$. Trivially, the eigenstates and eigenvalues are the EPR states $\psi_{ \pm}=1 / \sqrt{2}[\phi(1,6,7,8) \pm \phi(2,6,7,8)]$ and $E_{ \pm}=\epsilon_{4}+d+J \Delta \pm J / 2$, respectivelly. For the initial state considered, the probability to find the excitation on site 1 at time $t$ is given by $P_{\phi(1,6,7,8)}(t)=$ $\left\{1+\cos \left[\left(E_{+}-E_{-}\right) t\right]\right\} / 2$, while the probability to find it in 2 is $P_{\phi(2,6,7,8)}(t)=\left\{1-\cos \left[\left(E_{+}-E_{-}\right) t\right]\right\} / 2$.

At each instant of time $k \pi / 2 J$, where $k$ is an odd number, a state of maximal entanglement between the defects is obtained, $1 / \sqrt{2}[\phi(1,6,7,8) \pm \phi(2,6,7,8)]$. After a very long time, the states $\phi(1,6,7,8)$ and $\phi(2,6,7,8)$ finally start mixing with the other six registers (see Eq. (27)), where the cluster appears in a different position. The hybridization of these states in time is shown in Fig. 2 The top panel corresponds to a short time and the two lower panels are obtained after much longer times have passed.

Before allowing the system to evolve for a very long time, where the cluster delocalizes, states with concurrence very close to 1 can be created. We let the system evolve just up to one of the initial instants where a Bell state emerges. At this moment, the two defects can be quickly detuned and such maximally entangled state between the defects would be maintained for a long time until the cluster starts moving.

It is clear that if the initial state was one of the EPR states $1 / \sqrt{2}[\phi(1,6,7,8) \pm \phi(2,6,7,8)]$, it would take a very long time to change, since this would require moving the "heavy" cluster of three excitations together. The ability to keep the entanglement between the de- fects very large for a long time is a consequence of the large anisotropy of the $X X Z$ model. In this way, this result strongly differs from the dynamics considered in Ref. 19]. There, such effects of the Ising interaction are not seen, because the dynamics of correlations is studied for an $X Y$ model.
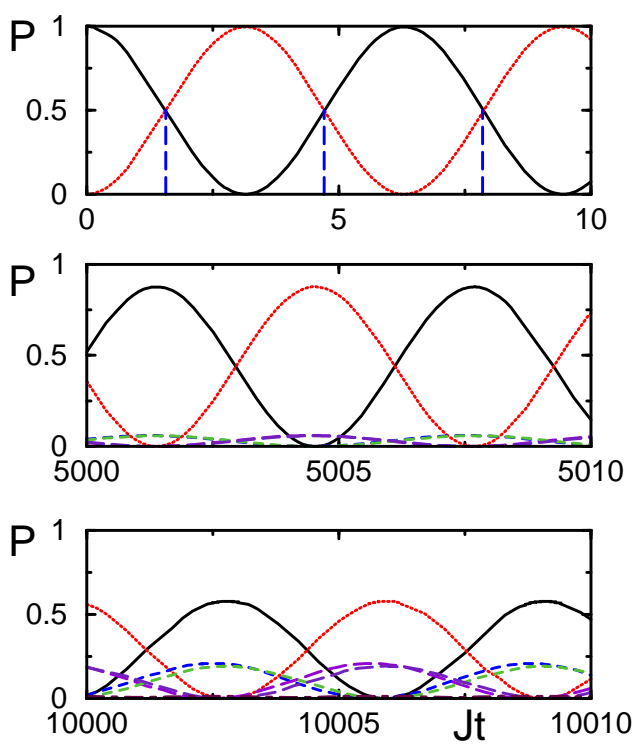

FIG. 2: (color online) We have a chain with 12 sites, 4 excitations, and two defects placed on sites 1 and 2. The parameters of the system are $d=10 J$ and $\Delta=50$. We choose $\phi(1,6,7,8)$ as the initial state and let the system evolve. The three panels show the probability for finding other quantum registers in time. For the short times of the top panel, $\phi(1,6,7,8)$ (black solid line) mixes only with $\phi(2,6,7,8)$ (red dotted line). The instants of time where Bell-type states are created are shown with vertical long dashed lines. They appear at $\pi / 2 J, 3 \pi / 2 J$, and $5 \pi / 2 J$. In the middle panel, maximally entangled states are not obtained anymore, as other quantum registers start mixing with the previous two. The probabilities for the registers $\phi(1,5,6,7)$ and $\phi(1,7,8,9)$ almost coincide and are shown with dashed lines (blue and green, respectively). The same happens to the registers $\phi(2,5,6,7)$ and $\phi(2,7,8,9)$, which are indicated with long-dashed lines (violet and indigo, respectively). The lower panel is obtained for times even longer, where the quantum registers $\phi(1,8,9,10)$ and $\phi(2,8,9,10)$ barely start appearing. They are shown in the figure with dot-dashed (maroon) lines, but can hardly been seen.

\section{B. Next nearest neighbor defects}

The description of the dependence of the maximum concurrence on the anisotropy coupling when the defects are next nearest neighbors is very complex, especially when several excitations are present. Just like in the end of the previous subsection, we restrict our study to the case of $\Delta \gg d / J$, where general remarks can be made.

The states with the largest concurrences are similar to the states given by Eq.(27). They have one excita- 
tion hopping between the two defects, though they are now separated, so the transition occurs in second order in $J$. The other excitations are bound together in clusters placed at least two sites away from the defects. Compared to the case of nearest neighbor defects, these remaining $N-1$ excitations have now one site less available, there are $L-7$ sites for them.

When $N-1$ is larger than $L-7$, the states with the largest concurrence have a different form. Sometimes these states can also have large concurrences, but this is not always the case, which explains the curves with low concurrence in the top and middle panels in Fig. [3]

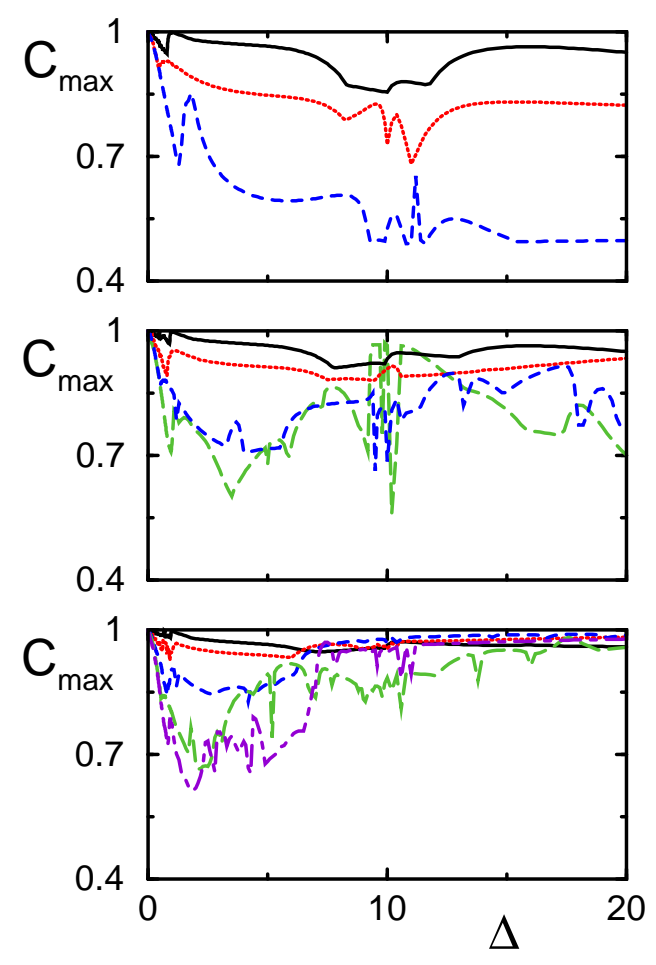

FIG. 3: (color online) Maximum concurrence vs. anisotropy coupling in closed Heisenberg spin chains with defects located on sites 1 and 3 . The energy difference between the defects and the other qubits is $d=10 \mathrm{~J}$. The panels correspond to data for chains of different lengths: $L=8$ (top), $L=10$ (middle) and $L=12$ (bottom). The maximum number of excitations considered for each chain is $L / 2$. The curves are chosen as follows: solid (black) curves for 2 excitations, dotted (red) for 3 excitations, dashed (blue) for 4 excitations, long dashed (green) for 5 excitations, and dot-dashed (violet) for 6 excitations.

The important result of this section is the verification that Bell-type states can again be obtained in the limit of large chains and $\Delta \gg d / J \gg 1$, even though the defects are separated.

An analysis for the time evolution of the system with a particular $d$-band quantum register taken as the initial state could again be made. It would be very similar to the one from the previous subsection, though a longer time is required for a maximally entangled state to appear.

\section{CONCLUSION}

We have shown how the interplay between interaction and disorder may affect the entanglement between two defects in a chain of qubits with anisotropic coupling. When the difference in energy between the defects and the ordinary qubits $d$ is much larger than the hopping integral $J$, several situations may be identified according to the value of the anisotropy coupling $\Delta$, the number of excitations in the chain, where the defects are placed, and the chain size.

The most general case refers to large chains and $\Delta \gg$ $d / J \gg 1$, where Bell-type states are obtained even for defects that are not nearest neighbors. These states have one excitation hopping between the two defects and all the others bound together in a cluster far from the defect sites. These clusters are a consequence of the large anisotropy. They move together and very slowly, allowing the maintenance of a large entanglement between the defects for a long time.

In the case of nearest neighbor defects, we also verify that even when the anisotropy coupling is not very large, the decrease in the value of entanglement between the defects is never very abrupt and it becomes less significant in larger chains. Analytical results were obtained for two excitations when $\Delta=0$ and $\Delta<d / J$. For $\Delta<d / J$ the state with the largest concurrence is the one with the smallest energy in its band.

We note that in this paper we have not developed any analysis in terms of quantum phase transition as done in several previous works [9, 10, 11]. By selecting two very large defects we guarantee that the entanglement between them is mostly kept very large. If only the $X X$ part of the Hamiltonian (1) was present, i. e. if $\Delta=0$, their concurrence would always have the maximum value 1, as seen from Figs. 11 and 3. It is the effect of the extra Ising interaction that can sometimes decrease the entanglement.

The present work has its clear relevance for quantum information and quantum computing, but it should also be of interest for condensed matter physics, where one wants to understand how localization may be affected by interaction and disorder.

\section{Acknowledgments}

L. F. S. acknowledges support by the NSF through grant No. ITR-0085922 and thanks M. I. Dykman for discussions. G. R. thanks FAPESP for funding this research. We are both very grateful to C. O. Escobar for helpful suggestions. 
[1] E. Schrödinger, Proc. Camb. Phil. Soc. 31, 555 (1935).

[2] M. A. Nielsen and I. L. Chuang, Quantum Computation and Quantum Information (Cambridge University Press, Cambridge, 2000).

[3] D. Bruß, J. Math. Phys. 43, 4237 (2002).

[4] S. Hill and W. K. Wootters, Phys. Rev. Lett. 78, 5022 (1997); W.K. Wootters, Phys. Rev. Lett. 80, 2245 (1998).

[5] M. I. Dykman and P. M. Platzman, Fortschr. Phys. 48, 9 (2000); P. M. Platzman and M. I. Dykman, Science 284 1967 (1999).

[6] X. Wang, Phys. Rev. A 64, 012313 (2001).

[7] L. F. Santos, Phys. Rev. A 67, 062306 (2003).

[8] L. F. Santos, M. I. Dykman, M. Shapiro, and F. M. Izrailev, Physical Review A 71, 012317 (2005); M. I. Dykman, F. M. Izrailev, L. F. Santos, and M. Shapiro, e-print cond-mat/0401201

[9] A. Osterloh, L. Amico, G. Falci, and R. Fazio, Nature (London) 416, 608 (2002).

[10] O. Osenda, Z. Huang, and S. Kais, Phys. Rev. A 67, 062321 (2003).

[11] Z. Huang, O. Osenda, and S. Kais, Phys. Lett. A 322,
137 (2004).

[12] G. Rigolin, Int. J. Quant. Inf. 2, 393 (2004).

[13] P. F. Kartsev and V. A. Kashurnikov, JETP Letters 80, 44 (2004).

[14] H. A. Bethe, Z. Phys. 71, 205 (1931); C. N. Yang and C. P. Yang, Phys. Rev. 150, 321, 327 (1966); M. Karbach and G. Müller, e-print cond-mat/9809162 F. C. Alcaraz, M. N. Barber, and M. T. Batchelor, Ann. of Phys. 182, 280 (1988).

[15] C. H. Bennett, H. J. Bernstein, S. Popescu, and B. Schumacher, Phys. Rev. A 53, 2046 (1996).

[16] L. F. Santos, J. Phys. A 37, 4723 (2004).

[17] L. F. Santos, G. Rigolin, and C. O. Escobar, Phys. Rev. A 69, 042304 (2004).

[18] L. F. Santos and M. I. Dykman, Phys. Rev B 68214410 (2003); M. I. Dykman and L. F. Santos, J. Phys. A 36, L561 (2003).

[19] L. Amico, A. Osterloh, F. Plastina, R. Fazio, and G. M. Palma, Phys. Rev. A 69, 022304 (2004). 